\title{
Optimal vaccine strategy to control COVID-19 pandemic in middle-income countries: Modelling case study of Thailand
}

Nantasit Luangasanatip

Mahidol Oxford Tropical Medicine Research Unit, Mahidol University

Wirichada Pan-Ngum

Mahidol Oxford Tropical Medicine Research Unit, Mahidol University

Juthamas Prawjaeng

Health Intervention and Technology Assessment Program, Ministry of Public Health

Sompob Saralamba

Mahidol Oxford Tropical Medicine Research Unit, Mahidol University

Lisa White

University of Oxford https://orcid.org/0000-0002-6523-185X

\section{Ricardo Aguas}

University of Oxford

Hannah Clapham

Saw Swee Hock School of Public Health, National University of Singapore

\section{Christopher Painter}

Health Intervention and Technology Assessment Program, Ministry of Public Health

Wang Yi

Saw Swee Hock School of Public Health, National University of Singapore

Wanrudee Isaranuwatchai ( $\boldsymbol{\nabla}$ wanrudee.i@hitap.net )

Health Intervention and Technology Assessment Program https://orcid.org/0000-0002-8368-6065

\section{Yot Teerawattananon}

Health Intervention and Technology Assessment Program, Ministry of Public Health

\section{Article}

Keywords: COVID-19, Coronavirus, vaccines, vaccination, middle-income countries, Thailand

Posted Date: February 24th, 2021

DOI: https://doi.org/10.21203/rs.3.rs-270635/v1 
License: (c) (i) This work is licensed under a Creative Commons Attribution 4.0 International License. Read Full License 
1 Optimal vaccine strategy to control COVID-19 pandemic in middle-income countries: Modelling case

2 study of Thailand

3

4 Nantasit Luangasanatip ${ }^{1}$, Wirichada Pan-Ngum ${ }^{1}$, Juthamas Prawjaeng ${ }^{2}$, Sompob Saralamba ${ }^{1}$, Lisa White ${ }^{3}$,

5 Ricardo Aguas ${ }^{3}$, Hannah Clapham ${ }^{4}$, Christopher Painter ${ }^{2}$, Wang $\mathrm{Yi}^{4}$, Wanrudee Isaranuwatchai ${ }^{2}$, Yot

6 Teerawattananon ${ }^{2,4}$

$7 \quad{ }^{1}$ Mahidol Oxford Tropical Medicine Research Unit, Mahidol University, Thailand

$8 \quad{ }^{2}$ Health Intervention and Technology Assessment Program, Ministry of Public Health, Thailand

$9 \quad{ }^{3}$ Nuffield Department of Medicine, University of Oxford, United Kingdom

$10 \quad{ }^{4}$ Saw Swee Hock School of Public Health, National University of Singapore, Singapore

11

12

13 Keywords: COVID-19, Coronavirus, vaccines, vaccination, middle-income countries, Thailand

14 Word count:

15 Main text: 2,729 words

16 Methods: 1,242 words 


\section{$1 \quad$ ABSTRACT}

2 Thailand is facing the dilemma of which groups to prioritise for the limited first tranche of vaccinations in

3 2021. A mathematical modelling analysis was performed to compare the potential short-term impact of

4 allocating the available doses to either the high-risk group (over 65-year-olds) or the high incidence group

5 (aged 20-39). Vaccinating the high incidence group with a vaccine with sufficiently high protection against

6 infection (more than 50\%) could provide enough herd effects to delay the expected epidemic peak,

7 resulting in fewer deaths within the 12-month time horizon compared to vaccinating the same number of

8 the high-risk group. After 12 months, if no further vaccination or other interventions were deployed, this

9 strategy would lead to more deaths. With the right vaccine efficacy profile, targeting the high incidence

10 groups could be a viable short-term component of the Thai vaccination strategy. These results and

11 emerging evidence on vaccines and susceptibility suggest prioritisation guidelines should be more

12 nuanced. 


\section{INTRODUCTION}

2 Towards the end of 2020, the first COVID-19 vaccines became available and this rapid development was

3 hailed as an unprecedented feat in science. However, many questions remain regarding the long-term

4 impact of the available vaccines, and their influence against new variants ${ }^{1-4}$. COVID-19 vaccines remain

5 scarce resources ${ }^{5}$, and it is unlikely that most countries without vaccine manufacturers will have sufficient

6 supplies of vaccines for their entire eligible population ${ }^{5-10}$. Furthermore, the global community does not

7 have the information to be sure what the most effective strategy is to use them to defeat COVID-19.

8 Evidence to support how best to allocate limited resources, for example which groups should be 9 prioritised for vaccination and which vaccines should be selected, would be useful to all countries that are 10 still awaiting vaccines.

12 The World Health Organization (WHO) has led the development of two major guidance documents for the 13 distribution and prioritisation of vaccine allocation within populations. The first is the Strategic Advisory 14 Group of Experts on Immunization's (SAGE's) Values Framework for the allocation and prioritisation of 15 COVID-19 vaccination that aims to ensure that successful COVID-19 vaccines are shared equitably among 16 and in countries ${ }^{11}$. The second guidance document is the Roadmap for prioritising population groups for

17 vaccines against COVID-19 ${ }^{12}$, focusing on prioritising subpopulations for vaccination in countries, based 18 on scenarios of vaccine supply and COVID-19 incidence. Despite these guidance documents, allocation 19 plans need to be contextualised as they are dependent on various factors, e.g., the country's population 20 demographics, underlying population health, local epidemiology of COVID-19 outbreak, health 21 infrastructure and staff, vaccine type and supply, and the current non-pharmaceutical interventions (NPIs) 22 implemented $^{13}$. 
1 Although Thailand was the second country to identify COVID-19 within their population ${ }^{14,15}$, the

2 implementation of NPIs in the first half of 2020 successfully suppressed local transmission throughout the

3 latter part of the year. The tourism sector is a vital part of the Thai economy ${ }^{16}$, which has suffered greatly

4 due to the NPIs in place (e.g., travel restrictions and quarantine requirements). Therefore, the potential

5 value of a COVID-19 vaccine in Thailand is different from many western countries where vaccines may

6 primarily be deployed to reduce morbidity and mortality. For Thailand, having COVID-19 vaccine may

7 allow the relaxation of certain NPIs in the country, allowing tourism and other sectors of the economy to

8 return to pre-COVID-19 normality. The context for COVID-19 vaccination in Thailand is quite specific.

9 Firstly, the number of available doses for the 12-month period starting from March 2021 is limited to approximately 31 million people (based on the current plan $)^{17}$. Secondly, the vast majority of the

11 population has not been exposed to the virus and, as of February 2021, Thailand has experienced few

12 cases $(<30,000)$ and deaths $(<100)$. Thus, if NPIs are relaxed after the vaccination campaign begins,

13 particularly international travel restrictions, we would expect a major epidemic peak to occur unless the

14 transmission blocking effect of the vaccines is as good as the summative effect of the NPIs that have been

15 in place. Unfortunately, there is a very high degree of uncertainty around the available vaccines'

16 transmission blocking effect ${ }^{18}$. Lastly, Thailand has a relatively small high-risk group with only $13 \%$ of the

17 population above the age of 65 . The high incidence group (ages 20-39), which is twice as large, is estimated

18 to have double the number of contacts per person.

20 This study shares a modelling experience in the Thai context to help identify the optimal vaccine strategy

21 (i.e., the group that it would be most beneficial to vaccinate) to control the COVID-19 pandemic for the

22 following year. We aim to examine the impact that COVID-19 vaccines with different types of efficacy can

23 have on different target populations, and to compare the trade-offs including to determine the efficacy

24 threshold for when the vaccine strategy may change within a predefined 12-month time horizon in which 
1 indirect effects may act to delay an epidemic. This analysis may be applicable to other countries with

2 similar characteristics and facing similar challenges regarding COVID-19, such as Vietnam, Laos, and many

3 small island countries who are heavily dependent on tourism and have low or no local transmission of

4 COVID-19.

5

6 RESULTS

7 The WHO defines COVID-19 vaccine efficacy to include 3 parts: 1) reducing susceptibility; 2) reducing

8 transmission from infected individuals (i.e., reduced infectivity); and 3) reducing severity ${ }^{19}$. As certain

9 characteristics of the vaccine remain uncertain, these analyses aimed to explore plausible scenarios of

10 different vaccine characteristics, including efficacy and protection duration. The target population that it

11 is most beneficial to vaccinate may vary depending on the type of efficacy that a vaccine has. The two

12 subpopulations that were considered for vaccination in these analyses were the high-risk population (the

13 elderly over 65 years old), and the high incidence group (20-39 years old) who have been most responsible

14 for transmission of COVID-19 in Thailand.

15

16 The three policy questions explored by the analyses are: 1) What is the impact of different types of vaccine

17 efficacy? 2) Is vaccine efficacy or protection duration a more preferable vaccine characteristic? 3) When

18 planning for vaccine allocation plan in Thailand, who should receive COVID-19 vaccines first? To answer

19 these questions, various scenarios were explored, and the characteristics of these analyses are described

20 in detail in the Methods section.

21 
1 Analysis 1: What is the impact of different types of vaccine efficacy?

2 Table 1 shows the results from the set of efficacy scenarios explored for Analysis 1, where vaccine efficacy

3 was assumed to be $70 \%$ for each type of efficacy and the protective duration was 1 year. The results

4 showed that, for both vaccination scenarios of the high-risk and high incidence groups, the type of efficacy

5 that reduced cases and deaths the most was when the vaccine efficacy reduced susceptibility. The vaccine

6 efficacy that was least desirable when vaccinating the high-risk group was reduced transmission, whereas

7 when vaccinating the high incidence group the least desirable efficacy type was reduced severity. The

8 results showed that total cases were similar in the scenario of no vaccination, compared to scenarios

9 where the vaccine reduced only severity for both vaccinated populations. The only scenarios where both

10 total cases and total deaths were substantially reduced compared to the no vaccination scenario, was

11 when the vaccine reduced susceptibility or transmission and was administered to the high incidence

12 population. When the high-risk group were vaccinated, only total deaths were reduced substantially, but

13 total cases remained similar to the no vaccination scenario.

14

16 characteristic?

17 For Analysis 2, scenarios were explored where the vaccine efficacy was varied between $70 \%$ and $90 \%$ and duration protection was varied between 6 months and 1 year. These scenarios were conducted for vaccinating both the high incidence and high-risk groups, and for reduced susceptibility and reduced severity vaccine efficacy types. For this analysis, we focused on reductions in susceptibility or severity as vaccine characteristics because the results from Analysis 1 showed that these were always preferable, in

22 terms of cases and deaths, to reduced transmission as a single characteristic of a vaccine. 
1 The results in Table 2 showed that when the high incidence group were vaccinated, a longer protection

2 duration with a lower efficacy was more effective at reducing cases and deaths than a shorter protection

3 duration and higher vaccine efficacy, when efficacy was the form of reduced susceptibility. This

4 relationship was also true in the scenario where the high-risk group were vaccinated although the

5 difference in results between the two scenarios was much smaller (Figure 1). The detailed breakdown of

6 results from this analysis are displayed in Table S1 in the Supplementary Materials.

8 For the analogous scenarios where the vaccine reduced severity, the results showed that for the high 9 incidence group the total number of cases were slightly greater for the lower efficacy, longer duration vaccine compared to the higher efficacy, shorter duration vaccine. However, the total number of deaths

11 in both scenarios were similar in both vaccine characteristic scenarios. For the high-risk group, the total

12 number of cases in both vaccine characteristic scenarios were similar, but the lower efficacy, longer 13 duration vaccine resulted in fewer deaths than the higher efficacy, shorter duration vaccine (Figure 2).

14 The detailed breakdown of these results can also be found in Table S2 in the Supplementary Materials.

15 For both types of efficacy explored, the results showed that a longer protection duration, of 12 months compared to 6 months, provided more benefit than the vaccine efficacy change from $70 \%$ to $90 \%$ in all

17 scenarios apart from when the high incidence group was vaccinated with a vaccine that reduced severity.

21 The results from Analysis 1 showed that in the case where a vaccine only had a single type of efficacy, the

22 high incidence group should be prioritised if the vaccine reduced susceptibility or transmission. However, 23 if the vaccine reduced severity then the high-risk group should be prioritised for vaccination. To further 
1 address this question, we considered scenarios where the vaccine had more than one types of efficacy. In

2 this analysis, different combinations of reduced susceptibility and reduced severity levels as a result of

3 vaccination were explored. If the prioritisation of groups for vaccination was based on the number of total

4 deaths, then the results showed that when reduction in severity from vaccination was either $70 \%$ or $90 \%$

5 and reduced susceptibility was $0 \%$, the preferred vaccine strategy was to prioritise the high-risk group.

6 However, when the reduction in susceptibility was greater than $60 \%$, then vaccinating the high incidence

7 group resulted in fewer deaths within 12 months of the roll out and more deaths if the time horizon is

8 extended (Figure 3).

9

10 Results are also presented from scenarios where the level of reduction in severity from vaccination was

11 fixed at $70 \%$ or $90 \%$ and the effect of vaccination on susceptibility is varied incrementally between $0 \%$ and

$1270 \%$ or $0 \%$ and $90 \%$, respectively (Figure S1 in the Supplementary Materials). The rationale for fixing the

13 reduction in severity and varying the reduction in susceptibility is that the evidence from clinical trials on

14 reductions in severity is established before evidence for reductions in susceptibility ${ }^{20}$.

16 DISCUSSION

17 This timely study improves informed choice for decision makers by providing the details, benefits, risks

18 and expected outcomes associated with each policy option. The findings also outlines the importance for

19 manufacturers, funders and researchers to develop more evidence regarding the impact of vaccines on

20 transmission, as the primary endpoints from the clinical trials for the available vaccines did not

21 satisfactorily address these policy-relevant questions ${ }^{20-25}$, which remain of paramount importance for

22 decision making. This study shows that reductions in susceptibility or transmission as a result of

23 vaccination is a hugely important characteristic for a country similar to Thailand (in term of factors such

24 as community transmission pattern, NPIs implemented, and risk of severe disease). Moreover, the type 
1 of vaccine efficacy is not the only important characteristic to distinguish between the available vaccines;

2 vaccine protection duration is an equally important consideration in order to maximise the population

3 health impact from the vaccination programme. Similarly, the duration of protection from the current

4 vaccines is not well-understood, post-vaccination surveillance can play a crucial role in determining this.

5 Manufacturers, researchers and governments should help the global community by closely monitoring

6 vaccine effectiveness of the initial vaccination cohorts in countries like Israel, the United Kingdom (UK)

7 and the United States that have implemented these vaccines already on a large scale ${ }^{26}$.

9 The WHO SAGE's prioritisation roadmap of COVID-19 vaccines ${ }^{12}$ would recommend that in the Thai context the high-risk group should be prioritised for vaccination, as a country with localised or limited

11 transmission. However, this study offers a different perspective by considering the potential benefits of

12 vaccinating alternative priority groups under various scenarios of characteristics of COVID-19 vaccine

13 efficacy. If a vaccine has an impact beyond just reductions in severity, this study shows that $60 \%$ or higher

14 percentage of reduction in transmission is pivotal in shifting prioritisation from the high-risk population

15 to the high incidence population, as they play a larger role in transmission in Thailand. There is now emerging evidence that vaccines may be able to reduce transmission (reduce infectivity) by up to $67 \%{ }^{27}$,

17 although the confidence interval for this estimate includes $0 \%$ so strategies which depend on this value 18 should be considered with caution. Given the results of our study and this new evidence, the WHO SAGE 19 and other authorities should reconsider the strategy of prioritising older populations over the younger

20 high incidence groups in populations with well-controlled community transmission, specific objectives and

21 time windows, and make their recommendations based on scenarios of vaccine characteristics and 22 coverage level combinations. 
1 Thailand's target vaccine supply is enough to vaccinate $50 \%$ of the population ${ }^{28}$ even though vaccine

2 nationalism (where richer nations are vaccinating their population with COVID-19 vaccines while

3 developing countries struggle to access supplies and have to wait ${ }^{29}$ ) may mean that Thailand receives less

4 than this target. Due to this limited supply, prioritisation of groups for vaccination is an unavoidable

5 decision for policymakers. The results of this study are being used for policy discussions in Thailand

6 regarding the prioritisation of groups for a COVID-19 vaccine amongst the general population. Health

7 professionals were already prioritised for vaccination, based on ethical justifications and in line with the

8 WHO SAGE's prioritisation roadmap of COVID-19 vaccines ${ }^{12}$. Although these decisions are ultimately left

9 to policymakers, it is important for researchers to produce evidence as presented in this study to inform

10 policy debates. Countries facing similar situations to Thailand should consider the applicability of this

11 evidence to their local context.

13 The results of this study must be interpreted with caution. Firstly, the mathematical model was matched

14 to Thai contract patterns and national COVID-19 outbreak data. Thus, the findings regarding the impact

15 of COVID-19 vaccination are most applicable to Thailand or other similar settings with well-controlled

16 community transmission. Countries with different demographic and outbreak profiles should ideally

17 conduct their own studies to contextualise evidence for informing policy. For example, Moore et al found

18 that prioritising the vaccination of the high-risk group is by far the most effective strategy for reducing the

19 number of deaths in the UK, where there is active local transmission, a greater proportion of the

20 population is high-risk group, and higher case-fatality rates ${ }^{30}$. Secondly, the vaccines under consideration

21 in this study were all hypothetical ones, so that the results of this study cannot be used to advocate for

22 individual vaccines that are currently available. The benefit of evaluating vaccines in this way is that we

23 considered a broader set of vaccine characteristics than the current evidence for real vaccines allows and

24 it will be more relevant to current policy questions as countries may be unable to rely solely on the supply 
1 of a single vaccine. This evidence may allow countries to have a tailored policy for selecting the most

2 appropriate vaccine for each population subgroup. Thirdly, we assumed equal vaccine effectiveness

3 across all population groups and did not consider vaccine side-effects and uptake, which may be a key

4 factor given that all available vaccines require two doses. Given the unique context of COVID-19, there is

5 not suitable evidence to justify an alternative vaccine uptake rate in Thailand. Nevertheless, the

6 importance of this parameter was recognised and will be reviewed when information becomes available

7 from the roll-out of the vaccination program. Fourthly, the impact of all NPIs were assumed to be constant

8 over time, but, in reality, they might vary over time. If this was the case, this would have implications for

9 the predictions for the results and conclusions of this study. Fifthly, the strategy of targeting the high

10 incidence group is critically contingent on further vaccination coverage in the following year; without this

11 continued coverage, the epidemic, delayed by partial herd immunity provided by a transmission blocking

12 vaccine, would lead to more deaths than the high-risk group strategy in the following year. Lastly, there

13 is a lot of individual parameter uncertainty in the model, both for vaccine-related parameters and COVID-

1419 clinical parameters. Both types of parameter should be subject to close monitoring as part of

15 vaccination programs to reduce this uncertainty. Many of these limitations should be addressed by

16 evidence generations activities by monitoring and evaluation systems. Inputs from this study could be

17 used to inform and develop these frameworks for monitoring and evaluation of COVID-19 vaccination

18 programs at national, regional and global levels.

\section{METHODS}

\section{Modelling structure}

22 In this study, we adapted an opensource compartmental age-structured model which is based on the SEIR

23 (Susceptible-Exposed-Infective-Recovered) structure developed by the COVID-19 International Modelling 
1 Consortium (CoMo consortium) ${ }^{31,32}$ (Figure S2 in Supplementary). In the model, the infected

2 compartments were stratified by symptoms, severity and treatment seeking and access. There are four

3 sub-compartments accounting for different severities of COVID-19 infection which are i) asymptomatic,

$4 \quad$ ii) mild to moderate symptomatic, iii) ICU and iv) ICU with ventilator.

5

$6 \quad$ Model parameters and outputs

7 The model uses publicly available country-specific data to define the population structure ${ }^{33}$ and mixing

8 contact patterns ${ }^{34}$ as model inputs. The model also uses publicly available country-specific data on cases

9 and mortality ${ }^{35}$ for visual calibration of model parameters to user-selected baseline scenarios. In this

10 study the model was calibrated against the data from the first wave of the pandemic in Thailand. In the

11 calibration, the reported daily new cases and deaths of COVID-19 infection were obtained from the

12 government's online data, which assumes that $100 \%$ of all cases were identified and reported ${ }^{36}$. We

13 believe that this assumption is reasonable as the first outbreak was mainly due to 3 clusters that occurred

14 during the middle of March and there were several months of no cases in Thailand after the initial

15 outbreak and no excess mortality ${ }^{35}$.

17 One other key set of parameters stratified by age was hospitalisation rates (AHR) and in-hospital fatality

18 rates (IFR), which were derived from previous published information from Wuhan, China and available

19 local data from Thailand from the 55 deaths up to $6^{\text {th }}$ June 2020 , respectively ${ }^{36}$. Virus-related parameters

20 including the incubation period, duration of symptomatic infection, risk of asymptomatic infection,

21 symptomatic infection (having clinical symptoms), admittance to ICU with and without a ventilator were

22 adopted from the values used in the CoMo model. Given the gap in knowledge on immunity acquired

23 post-infection, we assumed that natural immunity lasts an average of two years ${ }^{31,32}$ (See details in Table

24 S3 in Supplementary). The admission information for those with hospitalisation including the percentage 
1 of patients requiring ICU/ventilator, length of stay, and probability of death given ICU/ventilator were

2 derived from Thai data ${ }^{37}$. We used an 18 month time horizon for all model simulations, which allows for

3 a duration of 12 months post-vaccination. The primary model outputs were the number of COVID-19 cases

4 by severity, as well as deaths.

5

$6 \quad$ Non-pharmaceutical interventions (NPIs)

7 As the model calibration was based on the pandemic of the first wave, NPIs applied in Thailand included

8 hand hygiene and masking, social distancing, travel ban (or border closure) were tracked and incorporated

9 in the model (Figure S3 and Table S4 in Supplementary). As test, trace and isolation (TTI) varied spatially

10 and temporally, it was accounted for in the system dynamics via the force of infection, which changed

11 over time. The infection rate and effective coverage of each NPI including hand hygiene and social

12 distancing was estimated from visual calibration, by comparing the reported daily incidence and total

13 cases and deaths against the model prediction when the time for introduction of each NPI was clearly

14 stated. The baseline of $30 \%$ effective hand hygiene means reduction in the effective contact with infected

15 people by $30 \%$ while $40 \%$ social distancing means reduction in number of daily contacts across age groups

16 by $40 \%$. The set of estimated parameters given the optimal fit to the surveillance data from the first

17 outbreak during January to $6^{\text {th }}$ June 2020 was used in the baseline scenario (Figure S4 and Table S4 in

18 Supplementary).

19

20 Vaccine characteristics and implementation

21 The introduction of COVID-19 vaccination was expanded from the original CoMo model version 15.0,

22 where we defined the vaccine efficacy by 3 characteristics; i) reduced susceptibility (Efficacy 1), ii) reduced

23 transmissibility (Efficacy 2) and iii) reduced severity (Efficacy 3). Reduced susceptibility (Efficacy 1) means 
1 direct reduction in infection rate among vaccinated group while reduced transmissibility (Efficacy 2)

2 means reduction in force of infection among infections of vaccinated group and reduced severity (Efficacy

3 3) means reduced symptoms and progression to hospitalisation among those with vaccination. We

4 assumed that only one vaccine was used at a time, with a one dose regimen. The supply of vaccines is

5 likely to be limited for Thailand, we therefore assumed that it would be approximately 9 million doses in

6 2021. The assumed vaccine supply approximately corresponds to the size of the smallest potential group

7 for vaccination, the high-risk group (over 65 years old), who account for approximately 9 million people

8 in Thailand. This assumed vaccine supply allowed reasonable comparison between vaccinating this group

9 and the high incidence group (aged 20 to 39 years). It is further assumed that given the limited doses of vaccines, the Thai healthcare system would be capable of delivering the vaccine to reach the target

11 coverage within two months. As the protective duration of all the vaccines available is still unknown, we

12 assumed two scenarios, a short-term and long-term duration of protection of 0.5 or 1 year, respectively.

13 In the base case, a duration of protection of 1 year was assumed.

\section{Base case scenario}

Prior to the availability of the vaccine, there was assumed to be a small amount of local transmission in

17 the country spread from an original case upon day 1 in the model and no imported cases. To evaluate the

18 value of the vaccine, we assumed that following the roll-out of the vaccine there would be an accompanying relaxation of travel restrictions and quarantine measures to allow imported cases to enter the model. It was assumed that 100 cases of COVID-19 would be imported per day, based on expert

21 opinion from policymakers and representatives from the chamber of commerce. We assumed a constant

22 coverage of effective hand hygiene and facemask wearing of $30 \%$ among the population based on our

23 model visual calibration of the first outbreak. Social distancing measures were applied, which reduced

24 social contacts by $40 \%$ (excluding those at home, work and school). 
2 There were three main analyses to address the following questions: Analysis 1): What is the impact of

3 different types of vaccine efficacy? Analysis 2) Is vaccine efficacy or protection duration a more preferable

4 vaccine characteristic? Analysis 3: When planning for a vaccine allocation plan in Thailand, who should

5 receive COVID-19 vaccines first? Table 3 describes the combinations of scenarios that were explored for

6 each analysis.

7

8 Sensitivity analysis

9 The sensitivity analysis aimed to assess scenarios where the hypothetical vaccines have more than one 10 type of vaccine efficacy, and reduced severity from vaccination is fixed at $70 \%$ or $90 \%$ and the effect of 11 vaccination on susceptibility is varied incrementally between $0 \%$ and $70 \%$ or $0 \%$ and $90 \%$, respectively.

12 For each scenario, the percentage reduction of cases and deaths is compared to the base case where no 13 vaccines are administered. These sensitivity analyses were conducted for two scenarios of vaccine 14 protection durations of 6 months and 12 months, respectively. Additionally, these analyses were 15 conducted in scenarios where either the high-risk or high incidence groups were the vaccinated 16 population. The Figure S1 in supplementary materials also contain results from a sensitivity analysis where 17 there is a trade-off in vaccine efficacy between reduced susceptibility and reduced severity at a fixed level 18 of combined efficacy using the formula: $(1-$ overall efficacy $)=(1-$ efficacy $[1]) \times$

19 (1 - efficacy[3]). Reduced transmission from vaccination was not explored in sensitivity analyses as the 20 impact of reduced susceptibility is very similar to that of reduced transmission. The model code and the 21 data used in this paper can be found at https://github.com/slphyx/CoVac19TH. 
2 This project was funded by the Health Systems Research Institute (HSRI), the World Health Organization,

3 and the Wellcome Trust [Grant number 220211]. The Health Intervention and Technology Assessment

4 Program (HITAP) is funded by the Thailand Research Fund (TRF) under a grant for a Senior Research

5 Scholar (RTA5980011). HITAP is supported by the International Decision Support Initiative (iDSI) to provide

6 technical assistance on health intervention and technology assessment to governments in low- and

7 middle-income countries. iDSI is funded by the Bill \& Melinda Gates Foundation (OPP1202541), the UK's

8 Department for International Development, and the Rockefeller Foundation. HITAP is also supported by

9 the Access and Delivery Partnership (ADP), which is hosted by the United Nations Development

10 Programme (UNDP) and funded by the Government of Japan. The findings, interpretations and

11 conclusions expressed in this article do not necessarily reflect the views of the funding agencies.

12

13 Author contributions.

14 Conception of the work: NL, WP, JP, SS, LW, RA, CP, WY, WI, YT

15 Data analysis and interpretation: NL, WP, JP, SS, LW, RA, HC, CP, WY, WI, YT

16 Drafting the article: NL, WP, JP, SS, LW, CP, WI, YT

17 Revising the article: NL, WP, JP, SS, LW, RA, HC, CP, WY, WI, YT

18 Final approval of the article: NL, WP, JP, SS, LW, RA, HC, CP, WY, WI, YT

19

20 Competing interests.

21 The authors have no competing interests. 


\section{References}

21 Callaway, E. Could new COVID variants undermine vaccines? Labs scramble to find out,

$3 \quad<$ https://www.nature.com/articles/d41586-021-00031-0> (2021).

42 Nature. A review of 2020 through Nature's editorials. Nature 588, 537-538 (2020).

53 Else, $\mathrm{H}$. The science events to watch for in 2021. Nature.

64 Berkwits, M., Flanagin, A., Bauchner, H. \& Fontanarosa, P. B. The COVID-19 pandemic and the $7 \quad$ JAMA Network. Jama 324, 1159-1160 (2020).

85 So, A. D. \& Woo, J. Reserving coronavirus disease 2019 vaccines for global access: cross sectional $9 \quad$ analysis. bmj 371 (2020).

106 Purdum, T. S. Beware the danger of 'vaccine euphoria', $<$ https://www.statnews.com/2020/12/22/beware-the-danger-of-vaccine-euphoria/> (2020).

7 GAVI, CEPI \& World Health Organization. (World Health Organization,, 2020).

8 GAVI. COVAX Facility Portfolio: Probabilistic supply forecast (2020).

Tanne, J. H. Covid-19: US cases surge but vaccine distribution is slow. BMJ 372, doi:10.1136/bmj.n42 (2021). COVAX, <https://www.gavi.org/covax-facility> (2020). 19 vaccination, 14 September 2020. (World Health Organization, 2020). limited supply. World Health Organization (2020).

3 Wang, W. et al. Global, regional, and national estimates of target population sizes for covid-19 vaccination: descriptive study. bmj 371 (2020).

4 World Health Organization. Novel Coronavirus - Thailand (ex-China), $<$ https://www.who.int/csr/don/14-january-2020-novel-coronavirus-thailand-ex-china/en/> (2020).

15 Pongpirul, W. A., Pongpirul, K., Ratnarathon, A. C. \& Prasithsirikul, W. Journey of a Thai taxi driver and novel coronavirus. N Engl J Med 382, 1067-1068 (2020).

16 Bank of Thailand. Monetary Policy Report, $<$ https://www.bot.or.th/English/MonetaryPolicy/MonetPolicyComittee/MPR/BOX MRP/BOX M PR March2017 3.pdf> (2017).

17 Royal Thai goverment. National communicable disease committee's agreement on COVID-19 vaccine management and distribution strategy, $<$ https://www.thaigov.go.th/news/contents/details/38958> (2021).

18 Mallapaty, S. Can COVID vaccines stop transmission? Scientists race to find answers, <https://www.nature.com/articles/d41586-021-00450-z> (2021).

19 World Health Organization. WHO Target Product Profiles for COVID-19 Vaccines, <file:///C:/Users/acer/Desktop/who-target-product-profiles-for-covid-19-vaccines.pdf> (2020). Doshi, P. Pfizer and Moderna's "95\% effective" vaccines-we need more details and the raw data, <https://blogs.bmi.com/bmi/2021/01/04/peter-doshi-pfizer-and-modernas-95-effectivevaccines-we-need-more-details-and-the-raw-data/> (2021).

21 Polack, F. P. et al. Safety and efficacy of the BNT162b2 mRNA Covid-19 vaccine. N Engl J Med 383, 2603-2615 (2020).

22 Baden, L. R. et al. Efficacy and safety of the mRNA-1273 SARS-CoV-2 vaccine. N Engl J Med (2020). 
23 Voysey, M. et al. Safety and efficacy of the ChAdOx1 nCoV-19 vaccine (AZD1222) against SARSCoV-2: an interim analysis of four randomised controlled trials in Brazil, South Africa, and the UK. Lancet 397, 99-111 (2021).

24 Logunov, D. Y. et al. Safety and immunogenicity of an rAd26 and rAd5 vector-based heterologous prime-boost COVID-19 vaccine in two formulations: two open, non-randomised phase 1/2 studies from Russia. Lancet 396, 887-897 (2020).

25 Doshi, P. (British Medical Journal Publishing Group, 2020).

26 Bloomberg. More Than 134 Million Shots Given: Covid-19 Tracker, $<$ https://www.bloomberg.com/graphics/covid-vaccine-tracker-globaldistribution/?fbclid=IwAR02413sxE2P4Mc38W11593BISaoeNDYbD9M3hk2i3bsDQwuadYOWe94nU> (2021).

27 Voysey, M. et al. Single Dose Administration, And The Influence Of The Timing Of The Booster Dose On Immunogenicity and Efficacy Of ChAdOx1 nCoV-19 (AZD1222) Vaccine, $<$ https://papers.ssrn.com/sol3/papers.cfm?abstract id=3777268> (2021).

28 Thanthong-Knight, R. Thailand Aims to Inoculate At Least Half The Population in 2021, $<$ https://www.bloomberg.com/news/articles/2021-01-12/thailand-aims-to-inoculate-at-leasthalf-the-population-in-2021> (2021).

29 Kretchmer, H. Vaccine nationalism - and how it could affect us all, <https://www.weforum.org/agenda/2021/01/what-is-vaccine-nationalism-coronavirus-itsaffects-covid-19-pandemic/> (2021).

30 Moore, S., Hill, E. M., Dyson, L., Tildesley, M. \& Keeling, M. J. Modelling optimal vaccination strategy for SARS-CoV-2 in the UK. medRxiv (2020).

31 Linton, N. M. et al. Incubation period and other epidemiological characteristics of 2019 novel coronavirus infections with right truncation: a statistical analysis of publicly available case data. $J$ Clin Med 9, 538 (2020).

32 Khalili, M. et al. Epidemiological characteristics of COVID-19: a systematic review and metaanalysis. Plos One 148 (2020).

33 United Nations. Revision of world population prospects. (2019).

34 Prem, K., Cook, A. R. \& Jit, M. Projecting social contact matrices in 152 countries using contact surveys and demographic data. PLoS Comput Biol 13, e1005697 (2017).

35 Department of Disease Control of Thailand. COVID-19: Thailand Situation, $<$ https://ddc.moph.go.th/viralpneumonia/eng/index.php > (2021).

36 Nishiura, H. et al. Estimation of the asymptomatic ratio of novel coronavirus infections (COVID19). Int J Infect Dis 94, 154 (2020).

37 Petrilli, C. M. et al. Factors associated with hospitalization and critical illness among 4,103 patients with COVID-19 disease in New York City. MedRxiv (2020). 
1 Table 1. Comparison of impact of vaccines with different types of efficacy on clinical

2 outcomes when vaccinated with high incidence and high-risk groups.

\begin{tabular}{|c|c|c|c|c|c|c|c|}
\hline & $\begin{array}{l}\text { Vaccinated } \\
\text { population }\end{array}$ & Asymptomatic & $\begin{array}{c}\text { Mild- } \\
\text { moderate }\end{array}$ & $\begin{array}{c}\text { ICU } \\
\text { patients }\end{array}$ & $\begin{array}{l}\text { ICU with } \\
\text { ventilators }\end{array}$ & $\begin{array}{c}\text { Total } \\
\text { infection }\end{array}$ & Total deaths \\
\hline \multicolumn{8}{|c|}{ No Vaccine } \\
\hline $0-19$ & 0 & 123,000 & 368,000 & 78,000 & 52,000 & 621,000 & 0 \\
\hline $\begin{array}{l}20- \\
39\end{array}$ & 0 & 170,000 & 508,000 & 108,000 & 72,000 & 858,000 & 271 \\
\hline $\begin{array}{l}40- \\
64\end{array}$ & 0 & 204,000 & 610,000 & 130,000 & 87,000 & $1,031,000$ & 13,000 \\
\hline $65+$ & 0 & 28,000 & 83,000 & 18,000 & 12,000 & 140,000 & 25,000 \\
\hline Total & 0 & 525,000 & $1,569,000$ & 334,000 & 222,000 & $2,650,000$ & 38,000 \\
\hline \multicolumn{8}{|c|}{ Vaccinating high incidence: Efficacy 1 - Reducing susceptibility } \\
\hline 0-19 & 0 & 82,000 & 247,000 & 53,000 & 35,000 & 417,000 & 0 \\
\hline $\begin{array}{l}20- \\
39\end{array}$ & $9 \mathrm{M}$ & 91,000 & 275,000 & 57,000 & 38,000 & 462,000 & 161 \\
\hline $\begin{array}{l}40- \\
64\end{array}$ & 0 & 131,000 & 394,000 & 84,000 & 56,000 & 665,000 & 7,000 \\
\hline $65+$ & 0 & 17,000 & 51,000 & 11,000 & 7,000 & 86,000 & 14,000 \\
\hline Total & $9 \mathrm{M}$ & 321,000 & 968,000 & 205,000 & 136,000 & $1,630,000$ & 21,000 \\
\hline \multicolumn{8}{|c|}{ Vaccinating high incidence: Efficacy 2 - Reducing transmission } \\
\hline 0-19 & 0 & 80,000 & 242,000 & 52,000 & 34,000 & 408,000 & 0 \\
\hline $\begin{array}{c}20- \\
39\end{array}$ & $9 \mathrm{M}$ & 104,000 & 324,000 & 66,000 & 44,000 & 538,000 & 184 \\
\hline $\begin{array}{c}40- \\
64\end{array}$ & 0 & 128,000 & 387,000 & 82,000 & 55,000 & 652,000 & 7000 \\
\hline $65+$ & 0 & 17,000 & 49,000 & 10,000 & 7,000 & 84,000 & 13,000 \\
\hline Total & $9 \mathrm{M}$ & 329,000 & $1,002,000$ & 210,000 & 140,000 & $1,681,000$ & 21,000 \\
\hline \multicolumn{8}{|c|}{ Vaccinating high incidence: Efficacy 3 - Reducing severity } \\
\hline 0-19 & 0 & 123,000 & 368,000 & 78,000 & 52,000 & 622,000 & 0 \\
\hline $\begin{array}{l}20- \\
39\end{array}$ & $9 \mathrm{M}$ & 170,000 & 535,000 & 108,000 & 72,000 & 885,000 & 233 \\
\hline $\begin{array}{c}40- \\
64\end{array}$ & 0 & 204,000 & 611,000 & 130,000 & 87,000 & $1,032,000$ & 13,000 \\
\hline $65+$ & 0 & 28,000 & 83,000 & 18,000 & 12,000 & 140,000 & 25,000 \\
\hline Total & $9 \mathrm{M}$ & 525,000 & $1,598,000$ & 334,000 & 222,000 & $2,679,000$ & 38,000 \\
\hline \multicolumn{8}{|c|}{ Vaccinating high-risk: Efficacy 1 - Reducing susceptibility } \\
\hline $0-19$ & 0 & 122,000 & 365,000 & 78,000 & 52,000 & 616,000 & 0 \\
\hline $\begin{array}{l}20- \\
39\end{array}$ & 0 & 169,000 & 504,000 & 107,000 & 71,000 & 851,000 & 270 \\
\hline $\begin{array}{c}40- \\
64\end{array}$ & 0 & 202,000 & 605,000 & 129,000 & 86,000 & $1,021,000$ & 13,000 \\
\hline $65+$ & $9 \mathrm{M}$ & 17,000 & 55,000 & 11,000 & 7,000 & 90,000 & 15,000 \\
\hline Total & $9 \mathrm{M}$ & 510,000 & $1,528,000$ & 324,000 & 216,000 & $2,578,000$ & 28,000 \\
\hline
\end{tabular}




\begin{tabular}{|c|c|c|c|c|c|c|c|}
\hline & $\begin{array}{l}\text { Vaccinated } \\
\text { population }\end{array}$ & Asymptomatic & $\begin{array}{c}\text { Mild- } \\
\text { moderate }\end{array}$ & $\begin{array}{c}\text { ICU } \\
\text { patients }\end{array}$ & $\begin{array}{l}\text { ICU with } \\
\text { ventilators }\end{array}$ & $\begin{array}{c}\text { Total } \\
\text { infection }\end{array}$ & Total deaths \\
\hline $0-19$ & 0 & 122,000 & 365,000 & 78,000 & 52,000 & 616,000 & 0 \\
\hline $\begin{array}{l}20- \\
39\end{array}$ & 0 & 169,000 & 504,000 & 107,000 & 71,000 & 851,000 & 270 \\
\hline $\begin{array}{r}40- \\
64\end{array}$ & 0 & 202,000 & 604,000 & 129,000 & 86,000 & $1,021,000$ & 13,000 \\
\hline $65+$ & $9 \mathrm{M}$ & 27,000 & 88,000 & 17,000 & 11,000 & 143,000 & 24,000 \\
\hline Total & $9 M$ & 519,000 & $1,561,000$ & 330,000 & 220,000 & $2,630,000$ & 37,000 \\
\hline \multicolumn{8}{|c|}{ Vaccinating high-risk: Efficacy 3 - Reducing severity } \\
\hline $0-19$ & 0 & 123,000 & 368,000 & 78,000 & 52,000 & 622,000 & 0 \\
\hline $\begin{array}{l}20- \\
39\end{array}$ & 0 & 170,000 & 508,000 & 108,000 & 72,000 & 858,000 & 270 \\
\hline $\begin{array}{r}40- \\
64\end{array}$ & 0 & 204,000 & 611,000 & 130,000 & 87,000 & 1032,000 & 13,000 \\
\hline $65+$ & $9 \mathrm{M}$ & 28,000 & 92,000 & 18,000 & 12,000 & 149,000 & 16,000 \\
\hline Total & $9 \mathrm{M}$ & 525,000 & $1,579,000$ & 334,000 & 223,000 & $2,661,000$ & 29,000 \\
\hline
\end{tabular}

1

2 Note. ICU = intensive care unit. $\mathrm{M}=$ million people. This analysis assumed there was $30 \%$ hand hygiene 3 and $40 \%$ social distancing policy implemented. 
1 Table 2. Comparison of impact of vaccines efficacy and protection duration on clinical outcomes when 2 vaccinated with high incidence and high-risk groups.

3

\begin{tabular}{|l|c|c|c|}
\hline \multirow{3}{*}{$\begin{array}{l}\text { Population } \\
\text { vaccinated }\end{array}$} & Characteristic of vaccine & \multicolumn{2}{|c|}{ Efficacy type } \\
\cline { 3 - 4 } & & Reduced susceptibility & Reduced severity \\
\hline High incidence & Long duration, lower efficacy & 21,000 deaths, & 38,000 deaths, \\
& (1 year with 70\% efficacy) & $1,630,000$ cases & $2,679,000$ cases \\
\cline { 2 - 4 } & Short duration, higher efficacy & 24,000 deaths, & 38,000 deaths, \\
& (0.5 year with 90\% efficacy) & $1,858,000$ cases & $2,661,000$ cases \\
\hline High-risk & Long duration, lower efficacy & 28,000 deaths, & 29,000 deaths, \\
& (1 year with 70\% efficacy) & $2,578,000$ cases & $2,660,000$ cases \\
\cline { 2 - 4 } & Short duration, higher efficacy & 31,000 deaths, & 31,000 deaths, \\
& (0.5 year with 90\% efficacy) & $2,595,000$ cases & $2,657,000$ cases \\
\hline
\end{tabular}

4 
1 Table 3. Analysis characteristics

\begin{tabular}{|c|c|c|c|c|}
\hline Analysis & Vaccine characteristics & Vaccine duration & $\begin{array}{l}\text { Populations } \\
\text { vaccinated }\end{array}$ & NPIs \\
\hline 1 and 3 & $\begin{array}{ll}\text { - } & \text { Reduced susceptibility } \\
\text { - } & \text { Reduced transmission } \\
\text { - } & \text { Reduced severity } \\
\text { - } & \text { No vaccine }\end{array}$ & - 1 year & $\begin{array}{ll}- & \text { High-risk }(65+) \\
- & \text { High incidence } \\
& (20-39)\end{array}$ & \multirow{2}{*}{$\begin{array}{ll}\text { - } & 30 \% \text { hand } \\
\text { hygiene and } \\
\text { mask wearing } \\
\text { - } 40 \% \text { social } \\
\text { distancing } \\
\text { - } 100 \text { imported } \\
\text { cases per day }\end{array}$} \\
\hline 2 & $\begin{array}{ll}\text { - } & \text { Reduced susceptibility } \\
\text { - } & \text { Reduced transmission } \\
\text { - } & \text { Reduced severity }\end{array}$ & $\begin{array}{l}\text { - } 6 \text { months } \\
\text { - } 1 \text { year }\end{array}$ & $\begin{array}{ll}- & \text { High-risk }(65+) \\
- & \text { High incidence } \\
& (20-39)\end{array}$ & \\
\hline
\end{tabular}

2 
Figures

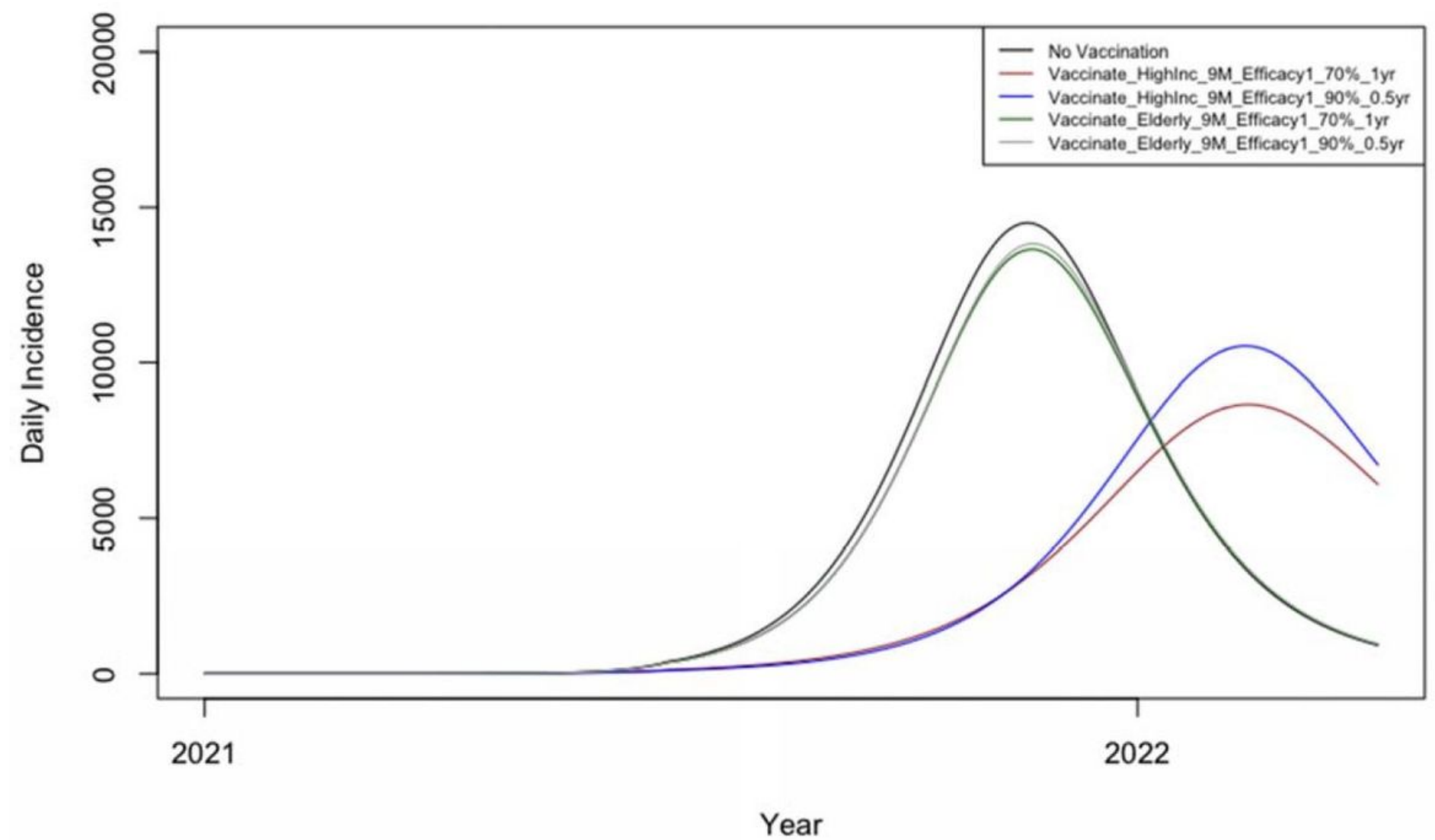

Figure 1

Comparison between vaccine efficacy to reduce susceptibility $70 \%$ with 1 year duration vs $90 \%$ with 0.5 year in both high incidence and high $\bigotimes$ risk groups 


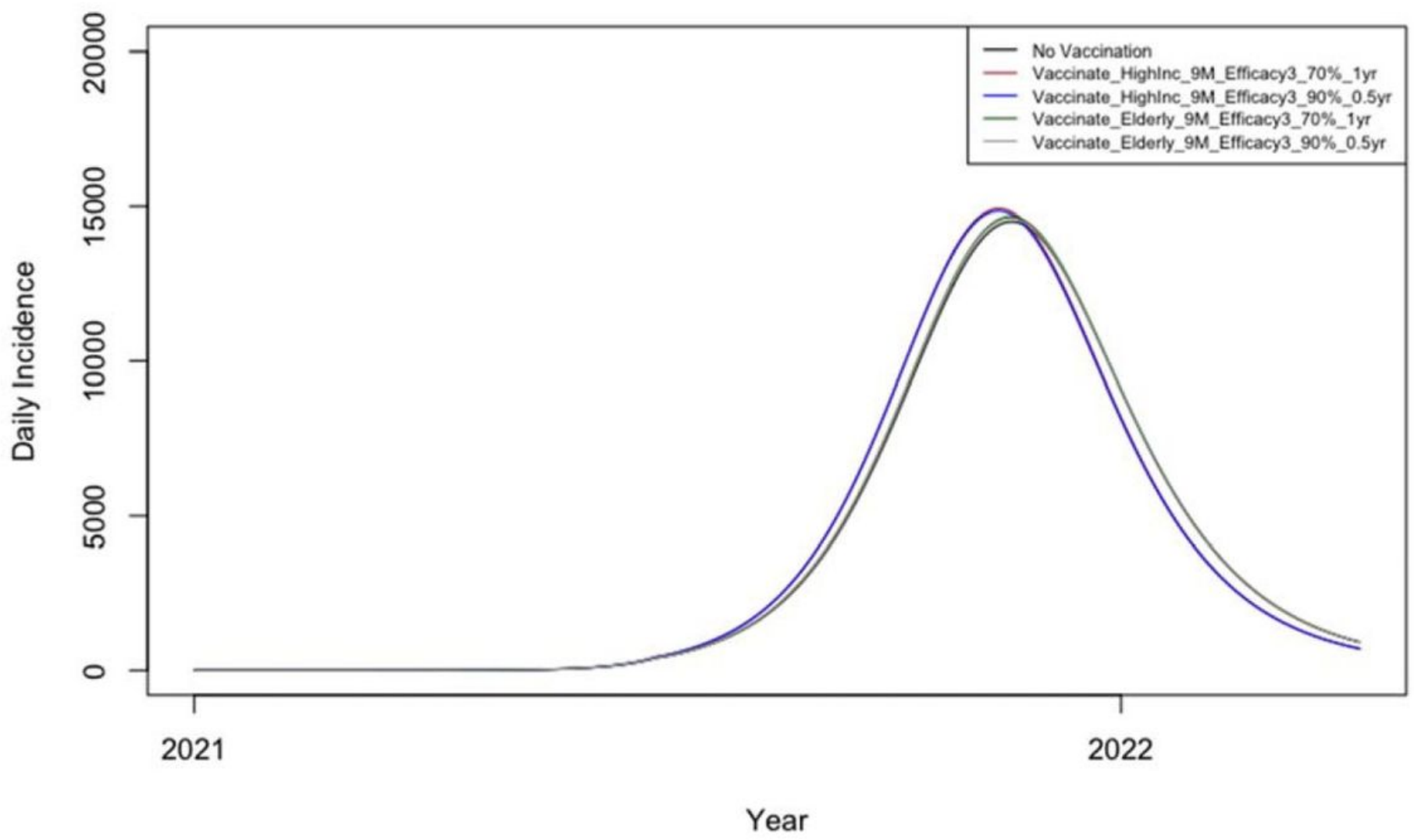

Figure 2

Comparison between vaccine efficacy to reduce severity at $70 \%$ with 1 year duration versus $90 \%$ with 0.5 year in both high incidence and high $囚$ risk groups 
$3 A$ ) Varied Efficacy1 (from $0 \%$ to $70 \%$ ) and varied Efficacy 3 (from $0 \%$ to $70 \%$ )

Overall $70 \%$ efficacy

\begin{tabular}{|c|c|c|c|c|}
\hline Vaccine duration 1 year & \multicolumn{2}{|c|}{ \%Death reduction } & \multicolumn{2}{|l|}{ \%Case reduction } \\
\hline Vaccine condition & Elderly & High Incidence & Elderly & High Incidence \\
\hline Eff1_0\% \& Eff3_70\% & $24.64 \%$ & $-0.14 \%$ & $-0.40 \%$ & $-1.09 \%$ \\
\hline Eff1_10\% \& Eff3_66.7\% & $24.92 \%$ & $3.74 \%$ & $0.04 \%$ & $2.59 \%$ \\
\hline Eff1_20\% \& Eff3_62.5\% & $25.15 \%$ & $8.28 \%$ & $0.48 \%$ & $6.74 \%$ \\
\hline Eff1_30\% \& Eff3_57.1\% & $25.37 \%$ & $13.73 \%$ & $0.93 \%$ & $11.48 \%$ \\
\hline Eff1_40\% \& Eff3_50\% & $25.65 \%$ & $20.10 \%$ & $1.37 \%$ & $16.93 \%$ \\
\hline Eff1_50\% \& Eff3_40\% & $25.90 \%$ & $27.41 \%$ & $1.81 \%$ & $23.23 \%$ \\
\hline Eff1_60\% \& Eff3_25\% & $26.18 \%$ & $35.77 \%$ & $2.25 \%$ & $30.44 \%$ \\
\hline Eff1_70\% \& Eff3_0\% & $26.48 \%$ & $44.64 \%$ & $2.70 \%$ & $38.47 \%$ \\
\hline Vaccine duration 0.5 year & \%Death reductic & & \%Case reductior & \\
\hline Vaccine condition & Elderly & High Incidence & Elderly & High Incidence \\
\hline Eff1_0\% \& Eff3_70\% & $15.01 \%$ & $0.21 \%$ & $-0.24 \%$ & $-0.31 \%$ \\
\hline Eff1_10\% \& Eff3_66.7\% & $15.83 \%$ & $2.84 \%$ & $0.02 \%$ & $1.84 \%$ \\
\hline Eff1_20\% \& Eff3_62.5\% & $16.61 \%$ & $5.93 \%$ & $0.29 \%$ & $4.16 \%$ \\
\hline Eff1_30\% \& Eff3_57.1\% & $17.37 \%$ & $9.39 \%$ & $0.56 \%$ & $6.72 \%$ \\
\hline Eff1_40\% \& Eff3_50\% & $18.20 \%$ & $13.53 \%$ & $0.83 \%$ & $9.58 \%$ \\
\hline Eff1_50\% \& Eff3_40\% & $18.91 \%$ & $18.40 \%$ & $1.10 \%$ & $12.81 \%$ \\
\hline Eff1_60\% \& Eff3_25\% & $19.77 \%$ & $24.02 \%$ & $1.38 \%$ & $16.50 \%$ \\
\hline Eff1_70\% \& Eff3_0\% & $20.55 \%$ & $30.38 \%$ & $1.65 \%$ & $20.70 \%$ \\
\hline
\end{tabular}

$3 B$ ) Varied Efficacy1 (from $0 \%$ to $90 \%$ ) and varied Efficacy3 (from $0 \%$ to $90 \%$ )

Overall $90 \%$ efficacy

\begin{tabular}{|c|c|c|c|c|}
\hline Vaccine duration 1 year & \multicolumn{2}{|c|}{$\%$ Death reduction } & \multicolumn{2}{|l|}{$\%$ Case reduction } \\
\hline Vaccine condition & Elderly & High Incidence & Elderly & High Incidence \\
\hline Eff1_0\% \&Eff3_90\% & $31.40 \%$ & $-0.27 \%$ & $-0.45 \%$ & $-1.26 \%$ \\
\hline Eff1_10\% \& Eff3_88.9\% & $31.67 \%$ & $3.56 \%$ & $-0.01 \%$ & $2.41 \%$ \\
\hline Eff1_20\% \& Eff3_87.5\% & $31.96 \%$ & $8.10 \%$ & $0.43 \%$ & $6.54 \%$ \\
\hline Eff1_30\% \& Eff3_85.7\% & $32.16 \%$ & $13.48 \%$ & $0.88 \%$ & $11.25 \%$ \\
\hline Eff1_40\% \& Eff3_83\% & $32.43 \%$ & $19.78 \%$ & $1.32 \%$ & $16.67 \%$ \\
\hline Eff1_50\% \& Eff3_80\% & $32.67 \%$ & $27.09 \%$ & $1.77 \%$ & $22.94 \%$ \\
\hline Eff1_60\% \& Eff3_75\% & $32.92 \%$ & $35.43 \%$ & $2.21 \%$ & $30.11 \%$ \\
\hline Eff1_70\% \& Eff3_66.7\% & $33.22 \%$ & $44.27 \%$ & $2.65 \%$ & $38.12 \%$ \\
\hline Eff1_80\% \& Eff3_50\% & $33.51 \%$ & $53.31 \%$ & $3.10 \%$ & $46.72 \%$ \\
\hline Eff1_90\% \& Eff3_0\% & $33.83 \%$ & $62.14 \%$ & $3.55 \%$ & $55.51 \%$ \\
\hline Vaccine duration 0.5 year & \multicolumn{2}{|c|}{ \%Death reduction } & \multicolumn{2}{|l|}{$\%$ Case reduction } \\
\hline Vaccine condition & Elderly & High Incidence & Elderly & High Incidence \\
\hline Eff1_0\%. \& Eff3_90\% & $19.09 \%$ & $0.05 \%$ & $-0.27 \%$ & $-0.41 \%$ \\
\hline Eff1_10\% \& Eff3_88.9\% & $19.28 \%$ & $2.43 \%$ & $-0.01 \%$ & $1.73 \%$ \\
\hline Eff1_20\% \& Eff3_87.5\% & $19.44 \%$ & $5.14 \%$ & $0.26 \%$ & $4.05 \%$ \\
\hline Eff1_30\% \& Eff3_85.7\% & $19.62 \%$ & $8.23 \%$ & $0.53 \%$ & $6.59 \%$ \\
\hline Eff1_40\% \& Eff3_83\% & $19.79 \%$ & $11.72 \%$ & $0.80 \%$ & $9.44 \%$ \\
\hline Eff1_50\% \& Eff3_80\% & $19.97 \%$ & $15.86 \%$ & $1.07 \%$ & $12.66 \%$ \\
\hline Eff1_60\% \& Eff3_75\% & $20.19 \%$ & $20.51 \%$ & $1.35 \%$ & $16.33 \%$ \\
\hline Eff1_70\% \& Eff3_66.7\% & $20.32 \%$ & $25.93 \%$ & $1.62 \%$ & $20.51 \%$ \\
\hline Eff1_80\% \& Eff3_50\% & $20.52 \%$ & $31.80 \%$ & $1.89 \%$ & $25.23 \%$ \\
\hline Eff1_90\% \& Eff3_0\% & $20.71 \%$ & $38.18 \%$ & $2.17 \%$ & $30.49 \%$ \\
\hline
\end{tabular}

\section{Figure 3}

Sensitivity analysis when varying the level of reduction in susceptibility (Efficacy 1 ) and varying the level of reduction in severity (Efficacy 3 ) at both $70 \%$ and $90 \%$ with different assumption of vaccine protective duration, 1 and 0.5 year 
This is a list of supplementary files associated with this preprint. Click to download.

- SupplementaryMaterialsCOVID19VaccineStrategiesThailand.pdf 